\title{
Occipital tACS bursts during a visual task impact ongoing neural oscillation power, coherence and LZW complexity
}

Authors:

Marta Castellano ${ }^{1}$, David Ibanez-Soria ${ }^{1}$, Eleni Kroupi ${ }^{1}$, Javier Acedo ${ }^{1}$, Michela Campolo ${ }^{2}$, Xenia Martinez ${ }^{4}$, Aureli SoriaFrisch $^{1}$, Josep Valls-Sole ${ }^{2}$, Ajay Verma ${ }^{3}$, Giulio Ruffini ${ }^{1,4}$

Affiliations:

1. Starlab Barcelona SL, Av. Tibidabo 47 bis. Barcelona, Spain

2. EMG Unit, Neurology Department, Hospital Clinic and IDIBAPS (Institut d'Investigació Augustí Pi i Sunyer), Facultat de Medicina, University of Barcelona. Barcelona, Spain

3. Biogen Inc. Cambridge MA 02142, USA

4. Neuroelectrics Corp., Cambridge, MA 02139, USA

Contact information: giulio.ruffini@neuroelectrics.com

Additional Title Page Footnotes:

- We introduce a bursting tACS protocol to study semi-concurrent tACS effects in the visual system and their impact on behavior as measured by reaction time.

- Burst $10 \mathrm{~Hz}$ tACS $\left(\right.$ tACS $_{10}$ ) applied to the visual cortex entrained $\gamma$-oscillations and increased RTs in a change-ofspeed detection visual task more than $70 \mathrm{~Hz}$ tACS $\left(\mathrm{tACS}_{70}\right)$ or Control conditions.

- Burst tACS 10 also decreased amplitude of the P300 peak, while increasing $\alpha$-power and $\gamma$-LZW complexity.

- Physiological and behavioral impact of occipital $\mathrm{tACS}_{10}$ and $\mathrm{tACS}_{70}$ was frequency-specific. $\mathrm{tACS}_{70}$ reduced $\mathrm{\gamma}$ oscillations after $20 \mathrm{~min}$ of tACS stimulation.

- Cognitive task may determine cortical excitation levels as measured by complexity metrics, as lower $\mathrm{Y}$-LZW complexity correlates to faster reaction times.

\section{SUMMARY:}

Little is known about the precise neural mechanisms by which tACS affects the human cortex. Current hypothesis suggest that transcranial current stimulation (tCS) can directly enhance ongoing brain oscillations and induce long-lasting effects through the activation of synaptic plasticity mechanisms [1]. Entrainment has been demonstrated in in-vitro studies, but its presence in non-invasive human studies is still under debate [2,3]. Here, we aim to investigate the immediate and shortterm effects of tACS bursts on the occipital cortex of participants engaged in a change-of-speed detection task, a task that has previously reported to have a clear physiology-behavior relationship, where trials with faster responses also have increased power in $\gamma$-oscillations $(50-80 \mathrm{~Hz})$ [4]. The dominant brain oscillations related to the visual task are modulated using multichannel tACS at 10 and $70 \mathrm{~Hz}$ within occipital cortex. We found that tACS stimulation at $10 \mathrm{~Hz}\left(\mathrm{tACS}_{10}\right)$ enhanced both $\alpha(8-13 \mathrm{~Hz})$ and $\gamma$ oscillations, in hand with an increase in reaction time (RT) in the change-of-speed detection visual task. On the other hand, tACS at $70 \mathrm{~Hz}$ desynchronized visual cortices, impairing both phase-locked and endogenous $\gamma$ power while increasing RT. While both tACS protocols seem to revert the relationship reported in [4], we argue that tACS produces a shift in attentional resources within visual cortex while leaving unaltered the resources required to conduct the task. This theory is supported by the fact that the correlation between fast RT and high $\gamma$-power trials is maintained for tACS sessions too. Finally, we measured cortical excitability by analyzing Event-Related-Potentials (ERP) Lempel-Ziv-Welch Complexity $(\mathrm{LZW})$. In control sessions we observe that lower $\mathrm{Y}$-LZW complexity correlates to faster reaction times. Both metrics are altered by tACS stimulation, as $\mathrm{tACS}_{10}$ decreased amplitude of the P300 peak, while increasing $\mathrm{V}$-LZW complexity. To this end, our study highlights the nonlinear cross-frequency interaction between exogenous stimulation and endogenous brain dynamics, and proposes the use of complexity metrics, as LZW, to characterize excitability patterns of cortical areas in a behaviorally relevant timescale. These insights will hopefully contribute to the design of adaptive and personalized tACS protocols where cortical excitability can be characterized through complexity metrics.

Keywords: tACS, entrainment, complexity, visual cortex. 


\section{INTRODUCTION}

A wide range of cognitive functions are mediated by the dynamic modulation of oscillatory activity within and between different brain regions. The alpha rhythm $(8-13 \mathrm{~Hz})$ has been associated to top-down processing [5,6], mediating attentional processes [7] and linked to functions such as working memory or visual perception. High frequency gamma rhythms $(30-80 \mathrm{~Hz})$ have been associated with feature binding [8], learning or attention [3]. Although such electrical field potentials reflect the balance of synaptic excitation and inhibition $[9,10]$, a clear association between cognitive process and brain dynamics remains unresolved. In particular, although correlations between cognitive processes and oscillation features have been revealed, causality remains an open question [11].

Dynamic structures of brain activity, such as correlations over many timescales or self-similarity, can be captured using nonlinear or algorithmic complexity estimation methods, such as the Lempel-Ziv-Welch complexity algorithm (LZW) [12]. The LZW algorithm provides an upper bound to the algorithmic or Kolmogorov algorithmic complexity exploiting repeating patterns in the data, and summarizes it using the underlying repeating string patterns $[13,14]$. Whether we adhere to an algorithmic information definition of complexity, or even if we use more classical entropic or fractal ones, we can ask what is the relationship of such metrics (derived from brain data) to behavior, health or conscious level, for example [9,10].

In turn, neuromodulatory transcranial electrical stimulation techniques such as transcranial current stimulation (tCS, also known as tES) allow for the modulation of brain activity and behavior [15] through the generation of weak electrical fields within a physiologically relevant range [16]. Transcranial current brain stimulation encloses a family of related noninvasive techniques which include direct current (tDCS), alternating current of sinusoidal form (tACS), and random noise current stimulation (tRNS). More generally, these techniques are based on the delivery of weak currents through the scalp (with electrode current intensity to area ratios of about 0.3-5 A/m) at low frequencies resulting in weak electrical fields in the brain (with amplitudes of about $0.2-2 \mathrm{~V} / \mathrm{m}$ ) $[17,18]$ ). Such electrical fields are unlikely to produce per se action potentials, but have an influence on the likelihood of neuronal firing by altering the transmembrane potential. The sub-threshold effects of tCS depend also on the temporal characteristics of the electrical stimuli, as simple models suggest frequency dependent sensitivity of individual neurons to tACS (resonance). The basic mechanism for interaction in tCS is thought to be the coupling of electrical fields to elongated neuronal populations such as pyramidal cells, where sub-threshold polarization occurs at the soma and along fibers. Physically, the external electrical field forces the displacement of intracellular ions (which move to cancel the intracellular electrical field), altering the neuron's internal charge distribution and modifying the transmembrane potential difference, which in turn affects firing rates and derived plastic effects. Realistic biophysical models of current propagation can be used to estimate [18] and optimize [19] the electrical fields generated by multichannel tCS montages.

Our motivation in this work is the vision that non-invasive tCS can be used to probe causal relations to link physiology with behavior, and that the immediate physiological effects of tCS as measured with EEG offer an unexplored window to study such relationships [20]. In the longer term, we believe that elucidating the interaction between non-invasive brain stimulation and endogenous brain activity - and behavior - will enable the development of individualized adaptive stimulation paradigms for clinical applications. In this study we aim to provide new evidence for frequency dependence of tACS effects in the visual cortex and further discern the functional role of oscillations in cognition.

While a multitude of studies address the clinical implications of these techniques [21,22], tCS provides also an opportunity to probe the causal role of brain oscillations in behavior. Several studies report behavioral enhancement after the application of tACS in processes like working memory [23] contrast sensitivity [3,24,25] or fluid intelligence [15]. Physiologically, behavioral changes after tACS have been associated to an enhancement of same-frequency oscillatory power. In hand with this enhancement of neuronal activity, tACS is thought to entrain spontaneous brain oscillations in the range of the stimulation frequency [26], as has been observed in-vitro [27]. However, the enhancement and entrainment of brain oscillations in humans appears to be state-dependent [27-29]. In fact, differences in endogenous oscillations alter the behavioral and electroencephalographic (EEG) effects of tACS as measured in the motor [27,30], prefrontal [15,31], and parieto-occipital cortex [32,33], where the response of brain activity is seen to depend non-linearly on the precise relationship between endogenous and exogenous dominant frequencies. In particular, the presence of entrainment and resonance depends on the dominant oscillatory activity of the network, as proposed by in-silico models, where entrainment can be observed in $10 \mathrm{~Hz}$-oscillating for tACS frequencies lower than $50 \mathrm{~Hz}$ [34]. In parallel, animal studies show that networks with pronounced endogenous activity impede the entrainment of activity patterns in-vitro within the tested frequency range $(0.5-2 \mathrm{~Hz})[27]$.

Here we aimed to test the relationship between observed visual cortex oscillations and behavior in a visual task, exploring in turn to what extent tACS can be used to enhance, entrain or otherwise modify endogenous oscillatory activity in healthy adults. To achieve this, we measured the behavioral and physiological impact of tACS in the occipital cortex through EEG 
while participants conducted a visual change-of-speed detection paradigm (see Figure $1 \mathrm{~A}$ ) as already used by other authors [4]. The task induces a reliable increase of $\gamma$-activity $(50-80 \mathrm{~Hz})$ in the early visual cortex, as well as a reduction of $\alpha$ (8-13 $\mathrm{Hz}$ ) and $\beta(13-25 \mathrm{~Hz})$ oscillations at both visual stimulus onset (VSO) and at the change-of-speed onset (CSO) [35,36]. Crucially, trials with faster response are associated with stronger $\gamma$-power [4], which establishes a clear if non-causal relationship between physiology ( $\gamma$-power) and behavior. With the objective of interacting with the specific dominant frequencies at particular intervals of the task [4,36], we designed a burst-tACS protocol that stimulates the occipital cortex during $5 \mathrm{~s}$ after VSO at 10 or $70 \mathrm{~Hz}$ frequencies ( $\mathrm{tACS}_{70}$ and $\mathrm{tACS}_{10}$ respectively, see Figure $1 \mathrm{~B}$ and $1 \mathrm{C}$ ).

To characterize tCS derived changes in brain dynamics, we propose the use of algorithmic complexity estimation metrics. To the best of our knowledge there are no studies on algorithmic complexity - as estimated by LZW - of brain oscillations under tACS. However, various authors have studied this metric in other scenarios. For instance, stroke patients, schizophrenia, and depression patients display higher LZW complexity on both a spontaneous and a cognitive task-related EEG activity compared to age-matched healthy controls (e.g., [37]). Spontaneous EEG complexity seems to decrease during anesthesia and NREM sleep. Casali et al. [38] found it decreased also in patients with Unresponsive Wakefulness Syndrome (UWS), Minimally Conscious State (MCS) or Emergence from MCS (EMCS), and in Locked-in syndrome (LIS). Also, complexity during mental arithmetic seems to decrease compared to rest in schizophrenia, depression and in healthy controls [37]. Some of these findings are also supported by MEG (magnetoencephalography) studies. MEG signals from schizophrenic patients seem to have higher LZW complexity compared to healthy controls [39], and depressed patients seemed to have higher MEG pre-treatment complexity that decreases after 6 months of pharmacological treatment [40]. Although MEG and EEG measure brain activity differently, it seems that their underlying complexity patterns follow a similar behavior. Other MEG studies have revealed decreased complexity in MEG signals in Alzheimer's patients compared to age-matched healthy controls [41], increasing complexity until the sixth decade of life in healthy subjects, and decreasing complexity after this age, as well as higher complexity in females compared to males [42]. A recent MEG study showed that LZW complexity increases during a psychedelic state of consciousness induced using ketamine, LSD, and psilocybin compared to a placebo effect [43].

Based on previous studies, we reasoned that $\mathrm{tACS}_{70}$ and $\mathrm{tACS}_{10}$ would have a differential impact on brain dynamics. First, we expected that $\mathrm{tACS}_{70}$ and $\mathrm{tACS}_{10}$ would interact with oscillatory activity at the visual cortex at those frequencies, reducing reaction times when enhancing $\gamma$ oscillations. Second, that entrainment, defined as end of stimulation phaselocked oscillations, should be observed after tACS bursts. Finally, that $t A C S_{70}$ and $t A C S_{10}$ would have a differential impact on brain complexity during stimulation.

\section{RESULTS}

In the results and posterior analysis we focus on the EEG bands and electrodes of interest: Alpha and Gamma at posterior electrodes, where the stimulation was targeted.

\section{(1) Reproducing the neural signature of the task with Control sessions}

The visual change-detection task has been linked to the activation of early visual areas, where it induces a reliable increase of $\gamma$-activity and reductions of alpha and beta oscillations at both the onset of visual stimulation (VSO) and at CSO (see Figure 1A) $[4,35,36]$. Consistent with previous reports, in Control sessions we observed a $\gamma$ power enhancement in occipital electrodes (Figure $2 \mathrm{C}$ ) at 0.2 seconds after CSO that decays around $0.4 \mathrm{~s}$. Alpha and Beta power display sustained reduction for longer latencies, co-occurring with the event-related potential (ERP) associated to CSO (Figure 2A). Taken together, these findings replicate the well-known neural signature associated with the processing of visual stimuli $[4,35,36]$.

\section{(2) tACS impacts CSO event related potentials}

Event Related Potentials (ERP) are widely used in cognitive studies as their peak amplitude is thought to reflect an alteration of cortical excitability reflecting a temporal realignment of neuronal activity to stimuli [44]. In particular, larger ERP peak amplitude reflects a greater temporal alignment of neural activity with a particular stimulus, and higher excitability $[10,45]$. The P300 component is produced by a distributed network of brain processes associated with stimulusdriven attention and memory operations and its amplitude and latency change as a function of cognitive resource allocation [44]. In this study, we examined the ERP that arises at the onset of change-of-speed (CSO) to test whether tACS interferes with visual excitability in a frequency-specific fashion (Figure 1A). We extracted the amplitude of the P300 associated to CSO (Figure 2A) and observed that in occipital electrodes the amplitude of the P300 is reduced with tACS 10 stimulation as compared to both $\operatorname{tACS}_{70}(p<0.05)$ and control sessions $(p<0.01)$ (Figure $\left.2 B\right)$ in the left-occipital cortex 
(electrode 01). Interestingly, this decrease in P300 amplitude in $\mathrm{tACS}_{10}$ sessions is also observed at P8, suggesting a possible alteration of the dorsal attention network.

\section{(3) tACS impacts endogenous oscillatory activity power (incoherent power analysis)}

To further study tACS modulation of ongoing activity, we examined whether tACS alters $\alpha$ and $\gamma$ power a 1s window shortly after each stimulation burst (tCS condition) and compared that to baseline (pre-EEG intervals, see Figure 1B). We extracted the power of oscillatory activity through the FFT, binned the frequency spectrum into bands of interest $(\alpha=[8,13]$ and $\gamma=[60$ 80] $\mathrm{Hz}$ ), and tested whether the tACS protocols modulated power at these bands in occipital electrodes through a Generalized Linear Mixed Model (GLMM, see Supporting Information for details). The GLMM revealed a significant interaction between tACS protocol and EEG-interval $(p<0.001$, $t$-value $=10.663)$. Further pairwise analysis of the model factors revealed a significant increase in $\alpha$-power at occipital electrodes after tACS $_{10}$ as compared to tACS 70 , while $\gamma$-power in occipital electrodes after $\mathrm{tACS}_{70}$ was lower as compared to tACS 10 (all comparisons $p<0.001$, see Figure 3B). Moreover, no significant change in $\gamma$-power in occipital electrodes after $\mathrm{tACS}_{70}$ in the post conditions was observed compared to Control sessions or to pre-EEG. Instead, $\gamma$-power seemed to decrease, as the stimulation blocks were finishing up (Figure 4A), hinting to a possible accumulative effect of tACS especially important at high stimulation frequencies.

One potential concern is that the observed power change due to $\mathrm{tACS}_{10}$ may to be due to an accumulative effect (plasticity) of tACS and not due to entrainment. To test for a possible interaction, we defined a GLMM where trial number is a fixed effect and found that the described effects are present in all 4 blocks of the task $(p<0.001)$.

Another potential concern is that this effect is not spatially localized to the tACS stimulation site (Figure 1C). To assess this, we defined a GLMM for parietal electrodes (P7 and P8). No statistical differences for $\alpha$-power across tACS protocols (control included) were found. Interestingly, $\gamma$-power after tACS $_{70}$ in parietal electrodes remained lower than $\gamma$-power after tACS $_{10}(p<0.001)$, as observed in occipital electrodes. This suggests that, while the modulation of $\alpha$-power was spatially localized at the stimulation electrodes, modulation of $\gamma$-power recruited other cortical areas out of the focus of stimulation.

To further investigate to what extent tACS modulates oscillatory activity in a lasting manner, we analyzed the EEG at postEEG intervals ( $3 \mathrm{~min}$ resting state intervals after the end of the behavioral task, see Figure 1B) and 15 min after that (postll EEG intervals). We defined a GLMM where tACS stimulation type and EEG-intervals are treated as fixed effects and explored their interaction (see model details in Supporting Information). The interaction between tACS stimulation type and EEG-interval was confirmed by a pairwise analysis of the GLMM factors, all of them significant with $p<0.001$.

Interestingly, we found that the $\alpha$ and $\gamma$-power alterations observed after tACS bursts in the tCS condition were maintained in both post and postII-EEG conditions (Figure $4 \mathrm{~A}$ ). In particular, the $\alpha$-power changes observed during tACS $_{10}$ were also observed in post and postII-EEG intervals (as compared to control and tACS $70, p<0.001$ ), replicating previous results reported in both bursting $[2,46]$ and continuous $\alpha$-tACS protocols $[27,33,47]$. Similarly, the $\gamma$-power increase observed during $\mathrm{tACS}_{10}$ stimulation was also observed in post and postII-EEG intervals (as compared to tACS ${ }_{70}$ and control, $\mathrm{p}<0.001$ ). Occipital $\gamma$-power in tACS $_{70}$ post sessions was statistically smaller than the $\gamma$-power observed in Control sessions. Pairwise analysis of GLMM factors revealed that occipital $\psi$-power during tACS/control sessions was larger than $\gamma$-power at post and postll intervals, and that $\alpha$-power during tACS/control sessions was smaller than $\alpha$-power at post and postll intervals), revealing the expected modulation of oscillatory activity by the task [35].

\section{(4) tACS impacts locked responses in time and frequency (coherent power analysis)}

In order to test whether tACS produces an entrainment effect on endogenous oscillations, we examined the responses of brain oscillations phase-locked to tACS burst in terms of its Stimulation Related Potential (SRP), the evoked potential that is expected to occur due to tCS. The tACS bursts were configured to end a zero phase (zero current). This time point defined the origin for stimulation phase locked EEG analysis, giving rise to Stimulation Related Potentials akin to ERPs.

SRP responses at occipital electrodes for all three stimulation protocols are displayed in Figure $3 \mathrm{~A}$ (between 0.25 and $1 \mathrm{~s}$ after tACS burst). Although no clear oscillations at the tACS stimulation frequencies are visible, we tested whether the oscillatory response phased-locked to the tACS burst (and thus, coherent) is modulated by the presence of tACS. For that, we extracted the complex FFT of oscillatory activity from the SRPs at occipital electrodes, binned the frequency spectrum into bands of interest $(\alpha=[8,13]$ and $\gamma=[60-80] \mathrm{Hz})$, averaged it across epochs, and finally computed its modulus. Gamma band tACS phase locked activity was reduced compared to baseline in tACS $_{70}$ sessions (t-test, $p=0.096$, Figure 3B), but increased in $\mathrm{tACS}_{10}$ sessions (t-test, $\mathrm{p}=0.076$ ) - both at trend level. In the control sessions, no change in the coherent $\gamma$ band power was observed ( $t$-test, $p=0.606$ ). Such changes in tACS-locked $\gamma$-power seem to increase further in tACS ${ }_{10}$ as 
bioRxiv preprint doi: https://doi.org/10.1101/198788; this version posted October 5, 2017. The copyright holder for this preprint (which was not certified by peer review) is the author/funder, who has granted bioRxiv a license to display the preprint in perpetuity. It is made available under aCC-BY 4.0 International license.

compared to $\mathrm{tACS}_{70}$ (rankusm u-test, $\mathrm{p}<0.01$, Figure 3B) in occipital electrodes. No differences in phase-locked $\alpha$-band are observed in any of the sessions.

\section{(5) tACS and LZW complexity}

As previously discussed, algorithmic complexity provides the means to study the structure of oscillatory brain dynamics beyond stationary methods based on spectral features. To further elucidate the manner in which tACS affects brain complexity, we estimated the LZW metric for the $\mathrm{tACS}_{10}, \mathrm{tACS}_{70}$ and control conditions using the EEG data after each tACS

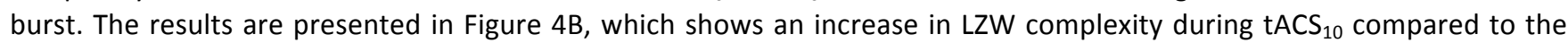
control condition in the high $\gamma$-band $(p<0.05)$.

\section{(6) Behavioral responses are altered during tACS}

During the visual task subjects were instructed to report the acceleration of an inward-moving grating (Figure $1 \mathrm{~A})$. We measured behavior through the analysis of the Reaction Time (RT) and the Percentage of Correct Responses (PC). The interaction between session and behavioral metrics was assessed with GLMM, where both subject and trial number were defined as random factors (see Supplemental Information). RTs of trials where tACS was active were significantly longer than observed in control trials $(p<0.001)$, as shown in Figure 1D. The slowing due to stimulation was observed in all the blocks of the task (interaction of tACS stimulation type and RT is significant in all the blocks, $p<0.001$ ), and is not tACSfrequency specific $(p=0.1741)$. Percentage of correct responses $\left(P C_{\text {control }}=97.6( \pm 0.4), P_{10 \mathrm{~Hz}}=96.8( \pm 0.5), P C_{70 \mathrm{~Hz}}=96.9( \pm\right.$ $0.7)$, mean \pm ste) was not altered by the presence of tACS stimulation ( $p>0.01)$. Note that, while RT seems to be altered by the presence of tACS, these differences cannot be uniquely attributed to a physiological impact of the tACS, as the sensory reports of participants exposed to active vs. control tACS sessions were different. In particular, $66 \%$ of participants reported feeling no stimulation in control sessions, in contrast to the $16 \%$ and $26 \%$ of participants who reported no-stimulation in $\mathrm{tACS}_{10}$ and $\mathrm{tACS}_{70}$ sessions respectively. Thus, control sessions cannot be considered sham (see Materials and Methods).

\section{(8) Predicting behavior from oscillatory activity}

To further understand the relationship between reaction time and its neural signature, we tested for the ability of oscillatory activity to predict behavioral responses (RT) as described in [4], where $\mathrm{y}$-band activity in the $50-80 \mathrm{~Hz}$ range of the calcarine sulcus was found to predict short reaction times in the particular visual change-detection task. This relationship was replicated in our control sessions, where the Pearson correlation between the $\gamma$-power and the RT displayed a significant correlation in occipital electrodes ( $r=-0.05, p<0.05$ two-tailed one-sample $t$-test). Next, we proceed to repeat the correlation analysis with $\mathrm{tACS}_{70}$ and $\mathrm{tACS}_{10}$ sessions. As in control sessions, $\mathrm{\gamma}$-power correlated with slower RT, maintaining the physiological relationship between $\gamma$-power and behavior. Interestingly, in tACS ${ }_{10}$ sessions, trials with higher power in low- $\gamma$ frequencies $(30-40 \mathrm{~Hz})$ at occipital electrodes predicted shorter RT, extending the frequency band that correlates with behavior reported in [35].

Regarding complexity metric as predictors of behavioral responses, there is a significant positive correlation between LZW complexity in the $\mathrm{\gamma}$-band and RT in control sessions, indicating that complexity in occipital cortex decreases with faster responses ( $p<0.05$ two-tailed one-sample t-test). Interestingly, tACS alters this relationship: no significant correlation appears at $\mathrm{tACS}_{70}$ sessions, while an increase in $\mathrm{\gamma}$-band complexity at $\mathrm{tACS}_{10}$ sessions predicts slower responses $(p<0.05$ two-tailed one-sample t-test). 


\section{DISCUSSION}

In this study, we examined first how tACS alters short-term physiology using a burst-EEG protocol that provides a small delay window to study concurrent tCS effects, and then how such effects are causally related to both secondary physiological effects and behavior, a technique already exploited with other stimulation techniques such as TMS [48]. tACS was used to probe the causal relationship between oscillatory activity within the occipital cortex and change-of-speed visual task, where subjects are instructed to detect a change on the acceleration of the visual stimuli (Figure $1 \mathrm{~A})$. This task produces a well-known and reproducible spectral signature in the visual cortex as assessed with MEG [4,35] and EEG [36], where it induces a reliable increase of $\gamma$-activity and reductions of alpha and beta oscillations at both VSO and CSO (see Figure 1A) $[4,35,36]$. A direct correlation with behavior was established in [4], where it was reported that $y$-band within occipital cortex $(60-80 \mathrm{~Hz})$ predicts shorter reaction times. Here, we interrogated this relationship between physiology and behavior by perturbing the visual system with tACS at 10 and $70 \mathrm{~Hz}$.

In control sessions, where no tACS was applied (0 currents), we reproduced the increase in $\gamma$-power with respect to baseline associated to shorter reaction times (RT), as reported in [4]. When stimulating with tACS ${ }_{10}, \gamma$-power increased even more but surprisingly this enhancement of $\gamma$ activity correlated with an increase of the RTs (slower responses), reversing the relation in the conventional control condition. This antagonistic relationship between physiology and behavior was observed in both coherent (i.e., phase-locked to the tACS cycle) and incoherent power analyses. While concurrent tACS 10 enhanced $\alpha$-oscillations $(8-13 \mathrm{~Hz})$, as previously reported in literature [26,27], in our study, tACS 10 also enhanced and entrained $\gamma$-oscillations. In addition, the concurrent $\alpha$-power increase due to tACS $_{10}$ in occipital electrodes was also observed in post and postII-EEEG intervals (as compared to control and tACS $_{70}, p<0.001$ ), replicating previous results reported in both bursting $[2,46]$ and continuous $\alpha$-tACS protocols $[27,33,49]$.

On the other hand, $\mathrm{tACS}_{70}$ stimulation had no statistically significant impact on concurrent $\mathrm{y}$-oscillations (neither incoherent or coherent power), while RT increased at the trend level. Surprisingly, tACS $_{70}$ reduced $\gamma$-power at post-EEG intervals, after the task and tACS stimulation had finished. The same trend was observed at the end of experimental blocks (60 trials), where $\gamma$-power seemed to decrease in $\mathrm{tACS}_{70}$ sessions (trend). This relationship appears to support the established relationship between physiology and behavior (at the trend level), where lower $\gamma$-power correlates with longer RT.

During control sessions, a decrease in $\gamma$-LZW (with respect to baseline) predicted shorter RTs. Stimulating with tACS 10 increased $\gamma-\mathrm{LZW}$, and the increase was associated to longer RTs. Our analysis establishes a relationship between a complexity metric and behavior that complements others observed in literature, where it is reported that LZW decreases in schizophrenia, depression and in healthy controls when the participants perform a mental arithmetic task compared to their resting state EEG [37]. While the precise neurophysiological interpretation of the complexity metrics needs further work, previous studies suggest that LZW is a non-linear estimator of cortical excitation [50]. In fact, the presence of the task itself without tACS (Control condition) already induced a change in brain complexity as compared to resting pre-EEG (Figure $4 C)$, suggesting that the presence of a cognitive task induces a change in the cortical dynamics that can be estimated through the analysis of complexity. A possible explanation for this relationship may be that when responses are fast (and correct) cortical circuits are more engaged in the task, and this restricts their dynamics to a particular reduced set of patterns, leading to a decrease in LZW. Since typically low complexity is related to more structure and predictability, it is expected to decrease when the brain dynamics are somehow synchronized with the repetitive pattern of the task. As such, $\mathrm{tACS}_{10}$ seems to make the visual system less structured and predictable, slowing task execution.

Regarding the ERP analysis, we found a decrease in ERP amplitude in tACS $_{10}$ sessions, which are associated to longer RT, according to what we would expect in literature. Indeed, ERP amplitude has been largely used as an index of cortical activation or excitability $[10,44]$ as peak amplitude of the different components of ERP are affected by a shift of attentional demands or perceptual load [51], and in particular, larger P300 amplitudes are associated with faster reaction times [52]. Modulations of ERP amplitude have been reported after DC electrical stimulation, an effect that is dependent on the stimulation polarity and duration [53]. In particular, a relationship with the efficacy of stimulation was established in [54] where a decrease in ERP amplitude for tDCS responders is reported. In relation to the change-of-speed detection task, our results show that burst $\mathrm{tACS}_{10}$ sessions decrease amplitude of the P300 peak, while increasing $\alpha$-power, $\gamma$-LZW complexity and RT, altogether indicating an underlying neural network misalignment to the stimulus or reduced activation.

Such relationship hint to the possibility that $\mathrm{tACS}_{10}$ increases inhibition in the visual cortex by enhancing $\alpha$ oscillations, ultimately reflecting a reduction of network alignment to visual stimuli [44]. These results are in agreement with recent studies that report a local encoding of visual stimuli and feedforward communication with higher cortical areas that are mediated by $\gamma$-band oscillations [4,5], while local $\alpha$-band oscillations $(8-13 \mathrm{~Hz})$ are involved in inhibitory feedback control processes [55] and long-range $\alpha$-oscillations that modulate feedback communication with distant areas [5,56]. In this line, behavioral tasks that emphasize top-down control of visual system display augmented synchronization in the low- 
bioRxiv preprint doi: https://doi.org/10.1101/198788; this version posted October 5, 2017. The copyright holder for this preprint (which was not certified by peer review) is the author/funder, who has granted bioRxiv a license to display the preprint in perpetuity. It is made available under aCC-BY 4.0 International license.

frequency bands [5,56], while local and intra-area synchronization in higher frequency hands $(\gamma$-band at $50-80 \mathrm{~Hz})$ is reported in tasks that mostly recruit feed-forward communication $[5,35,57]$. In contrast, changes in $\gamma$-band oscillations induced by tACS $_{10}$ (both phase-locked to the tACS cycle or incoherent to tACS) do not seem to alter the relation between $\gamma$ power and behavior, as in all tACS sessions trials, higher $\gamma$ power correlated with shorter reaction times. The physiological changes induced by tACS may either impact the local encoding of the visual task or produce a shift in attentional resources due to the presence of tACS itself. Such difference determines whether changes in cortical dynamics imply a direct interaction with cortical dynamics or an indirect modulation due to sensory modulations. While participants were able to identify control conditions, no sensory differences were reported for the two different stimulation frequencies (tACS $_{10}$ or $\left.\mathrm{tACS}_{70}\right)$, as evaluated by secondary effects questionnaires. Until further studies are conducted to understand sensory differences associated to tACS, we can argue for a frequency-specific modulation of cortical dynamics due to tACS.

\section{CONCLUSIONS}

The study of high-level cognitive processes may benefit from non-invasive transcranial alternating current stimulation (tACS) as a method to interrogate causal relationships between physiology and behavior. Future advancements will provide an improved understanding of the particular relation between endogenous and tACS-induced oscillations, allowing the modulation of the endogenous oscillations relevant to the cognitive function of interest. Such interaction, however, can take several forms (up or down-regulation of endogenous oscillations or phase-locking) and the effects on specific brain dynamical aspects are starting to be elucidated like in this study - where we report differential effects of tACS 10 and tACS 70 on the physiological response of the visual cortex. As to study differential impact of brain dynamics by tACS and cognitive task, we introduce algorithmic complexity estimating metrics (such as LZW). As argued elsewhere by us and others, such metrics should characterize the computational characteristics of cortical circuitry as it engages with the external world [9] and should be related to power-law behavior of recorded signals, which in turn are believed to be linked to neuronal excitation inhibition balance $[10,13]$. Our vision is that complexity metrics, as LZW, will enable the characterization of the excitability patterns of cortical areas in a timescale that allows to quantify excitability changes induced by the presence of cognitive tasks. 


\section{MATERIALS AND METHODS}

Participants: Thirty healthy subjects (mean age of $26.6 \pm 4.9$ years, 13 male) participated in a randomized, double-blinded study, with a within-subject design. Three randomized recording sessions (control, tACS 10 and tACS to $_{7}$ ) were separated by a 1 week of washout period to avoid carryover effects [58]. Participants gave written informed consent and received compensation for their participation. No history of neurological or psychiatric disorders was reported or any other contraindication to tCS [58]. The experimental campaign was conducted at Hospital Clinic and approved by the ethics research committee before start.

Experimental procedure: After the placement of the tACS and EEG electrodes, participants were familiarized with the behavioral task. The experiment started with the recording of $3 \mathrm{~min}$ of eyes-open rest EEG (while gazing at a fixation point), 3 min of eyes-closed rest EEG and continued with the recording of 240 trials of the behavioral task. The task was organized in 4 blocks of 60 trials each, with 5-15 min breaks across blocks so that the subjects could rest. Sessions were closed with 3 min of resting-state EEG (during fixation) and $3 \mathrm{~min}$ of eyes-closed rest EEG at the end of the behavioral task (post-EEG) and 15 min later (postII-EEG).

tACS protocol and EEG recording: Transcranial alternating current stimulation (tACS) was applied via three gelled $\mathrm{Ag} / \mathrm{AgCl}$ electrodes of $\pi \mathrm{cm}^{2}$ size (Pitrodes used with Starstim, Neuroelectrics) located at PO3, PO4 and Oz, placed according to a multi-electrode montage optimized for stimulation at BA17 using a realistic head model (Stimweaver, [19]). In the active conditions, AC currents were applied with $1.2 \mathrm{~mA}$ intensity (the cortically normal component of the electric field distribution is shown in Figure $1 \mathrm{C}$ ) in 5 -sec long bursts beginning and ending a zero phase. During control sessions zero current stimulation was delivered. The stimulation device was forced to end each tACS stimulation cycle at phase 0 . For this reason, we adjusted the frequencies $\alpha$ and $\gamma$ to $10.17 \mathrm{~Hz}$ and $69.99 \mathrm{~Hz}$ frequencies respectively. In each session, a total of 240 stimulation bursts were delivered at VSO (Figure $1 \mathrm{~A}$ ), adding up to a total stimulation time of 20 minutes. All subjects reported that stimulation did not induce phosphenes. Note that the intervals between tACS bursts were varied depending on the participant's response to the cognitive task, so no phase-synchrony was enforced between tACS bursts. EEG was corecorded in 5 electrodes located in parieto-occipital electrodes throughout the duration of the experiment (Figure 1B) with the same electrode type as tACS. EEG signals were recorded at $500 \mathrm{~S} / \mathrm{s}$ (24 bit) with the same device providing tACS (Starstim, Neuroelectrics). Electrode impedance was kept below $10 \mathrm{k} \Omega$, and the electrical reference placed at the earlobe.

Participants completed a questionnaire to assess the presence of visual and skin sensations after every session [58]. Sensations reported during active tACS were a mild itching sensation (33.3\% of the subjects), followed by a moderate sleepiness (25.6\%) and a mild discomfort sensation under the stimulation electrodes (6.67\%), in a comparable range of those reported in literature [58,59]. A single participant reported nausea after tACS (2.2\% prevalence). Similar effects were also reported during the control session, as $7.78 \%$ of participants reported a mild itching sensation, $12.2 \%$ reported moderate sleepiness and a subject mild discomfort sensation under the stimulation electrodes. Finally, the $33.3 \%$ of the participants thought they were being stimulated in the control sessions, while $84 \%$ and $74 \%$ of participants reported stimulation in $\mathrm{AACS}_{10}$ and $\mathrm{tACS}$ 70 sessions respectively.

Behavioral task: Participants were required to respond with a keypress to the change-of-speed of an inward-moving visual stimulus (visual change-detection) in a reaction time visual task paradigm $[4,35,36]$. Each trial began with the display of a fixation point (Gaussian of diameter $0.5^{\circ}$ ), and subjects were instructed to fixate to that position through the length of the trial. After 1 to 1.5 seconds (interval randomly chosen from a uniform distribution of 1-1.5 s) the fixation point was replaced by a moving grating (sine wave of $5^{\circ}$ located at the fovea contracting towards the fixation point at a spatial frequency of 4 cycles $/{ }^{\circ}$, contrast 100\%). After 6 to 8 seconds, its velocity increased to $2.2 \mathrm{deg} / \mathrm{s}$ until response was reported or 0.8 seconds passed $(\mathrm{CH}$ onset, see Figure $1 \mathrm{~A})$. Subjects were instructed to report the velocity increase with a button press on a keyboard, which made the moving grating disappear. Feedback was provided to participants via OK/KO signs after their response. A response earlier than 0.2 seconds after CSO was reported as KO. Stimuli were displayed on an LCD screen located at $60 \mathrm{~cm}$ of the subject, with a vertical refresh rate of $60 \mathrm{~Hz}$.

Data analysis: The analysis was performed using customized Matlab code (MathWorks Inc. Natick, MA, USA), EEGlab [60] and FieldTrip [61].

Behavioral analysis: subject responses were quantified in terms of the percentage of correct responses (i.e., correct detection of the velocity change or PC) and the reaction time (RT, i.e. delay in ms between $\mathrm{CH}$ onset and the keypress). Sessions whose PC was smaller than $85 \%$ were rejected. The differences in RT and PC were quantified by a generalized linear mixed-effects regression model (GLMM), see Supplemental Information. 
EEG analysis: raw EEG was cut into epochs of interest depending on whether we would analyse time-locked responses or ongoing oscillatory response. To assess visual cortex responses, we analyzed the signal of occipital electrodes 01 and 02. All epochs were filtered as follows: epochs were individually transformed into the spectral domain using the direct FFT transformation. Bins not in the frequency ranges of $[60,80]$ and $[5,40] \mathrm{Hz}$ were removed and subsequently returning to the temporal domain applying the inverse Fourier transform. Epochs containing signals with amplitude out of the $+/-50 u V$ range were rejected (high amplitude threshold) after filtering. Finally, unless indicated, EEG signals were referenced to the Pz electrode.

Subject rejection: After artifact correction and filtering, subjects containing less than 60 artifact-free 1 s epochs (at any electrode) were rejected, as a compromise between a sufficient number of epochs and subjects for a significant statistical analysis. As result, 7 subjects out of the 30 were discarded from further analysis.

Time-locked responses: Two different events in the task were used to study time-locked responses. tACS-burst EEG epochs were $1 \mathrm{~s}$ long starting $250 \mathrm{~ms}$ after the end of tACS stimulation (see Figure 1A). Residual amplitude clipping observed at the end of tACS bursts was corrected before the filtering step as described in Supporting Information. Subject's stimulation related responses (SRPs) was calculated averaging occipital electrodes epochs in the time domain. Also, epochs of [-0.2, 1] $\mathrm{s}$ around the CSO were extracted (Figure 1A) for ERP and spectral analysis in occipital electrodes.

Ongoing oscillatory responses: The analysis of ongoing oscillatory responses largely followed earlier work [26,46,62]. Resting state intervals in pre-EEG, post EEG and postII-EEG intervals (Figure 1B), were segmented into 1s epochs. During behavioral task, (Figure 1A), 1s epochs starting $250 \mathrm{~ms}$ from the end of the tACS were extracted.

Spectral analysis: Two different methodologies were used to conduct spectral analysis. On the one hand, epochs around CSO were analyzed for non-phase-locked oscillation by means of the time-frequency representations (TFRs) as described in [35]. Briefly, TFRs of frequencies between $30-100 \mathrm{~Hz}$ were obtained using the multi-tapering method, in steps of $2.5 \mathrm{~Hz}$, while TFRs of frequencies between $5-30 \mathrm{~Hz}$ were computed using a wavelet transform. The TFR were computed using a Fourier transform on $1 \mathrm{~s}$ epoched data in $50 \mathrm{~ms}$ steps. A smoothing of $\pm 5 \mathrm{~Hz}$ (squared function) was applied around each center frequency as reported in [4,5]. On the other hand, SRP power (coherent power) was calculated at $\theta=[5,8] \mathrm{Hz}$, $\alpha=[8,13] \mathrm{Hz}, \beta=[13,25] \mathrm{Hz}$ low $-\gamma=[30-40] \mathrm{Hz}$ and $\gamma=[60-80] \mathrm{Hz}$ bands via trapezoidal integration of the power spectral density of SRP. Calculated power is referenced (divided) to band power calculated the pre-EEG-eyes open epochs.

Complexity analysis: As described in [12,13], in LZW we consider a string of characters and alphabet with A symbols (typically binary) of length $\mathrm{n}$. The algorithm works by initializing the dictionary to contain all strings of length one and then it scans through the input string sequentially until it finds a string that does not belong to the dictionary, and adds it to the dictionary. This process is repeated until all input string has been scanned through. Following this process we end up with a set of words $c(n)$ that make up the dictionary. The length of the compressed string is lengthLZW <= $n$ (an upper bound to Kolmogorov or algorithmic complexity). The description length of the sequence encoded by LZW would have length equal to the number of phrases times the number of bits needed to identify a seen phrase plus the bits to specify a new symbol (to form a new phrase), hence (1) lengthLZW $=c(n) \log 2[c(n)+\log 2 A] \sim c(n) \log 2[c(n)]$. The lengthLZW is normalized by the original string length. The input string is binary and is derived by taking the median of the input time series as the threshold as it is a robust metric against outliers, assigning zeros to all values below the threshold and ones to all values above the threshold. The input time series consist of 4 -sec epochs filtered in the $\theta=[5,8] \mathrm{Hz}, \alpha=[8,13] \mathrm{Hz}, \beta=[13,25] \mathrm{Hz}$ low- $\gamma=[30-40]$ $\mathrm{Hz}$ and $\mathrm{\gamma}=[60-80] \mathrm{Hz}$ bands for each electrode.

\section{AUTHOR CONTRIBUTIONS}

DI, MC, MCam, XM conducted the data recording and MC, DI, EK and ASF did the analysis. MC and GR co-designed the protocol, coordinated the data analysis. GR and EK prepared the code for LZW analysis. GR designed the stimulation montage. JV coordinated data recording and MC, DI, EK, ASF, JV, AV and GR helped write the manuscript.

\section{ACKNOWLEDGMENTS}

This work was partly funded by Biogen Pharma. The complexity analysis part was partly been undertaken under the umbrella of the European FET Open project Luminous. This project has received funding from the European Union's Horizon 2020 research and innovation program under grant agreement No 686764.

\section{Conflict of interest}


bioRxiv preprint doi: https://doi.org/10.1101/198788; this version posted October 5, 2017. The copyright holder for this preprint (which was

not certified by peer review) is the author/funder, who has granted bioRxiv a license to display the preprint in perpetuity. It is made available under aCC-BY 4.0 International license.

GR is a co-founder, shareholder and employee of Neuroelectrics, the company that provides Starstim and Stimweaver. MC, EK, DI, XM and ASF, are employees of Starlab, the company that gave birth to Neuroelectrics in 2011. All other authors declare no potential conflicts of interest with respect to the research, authorship, or publication of this article. 


\section{FIGURES AND TABLES}

A
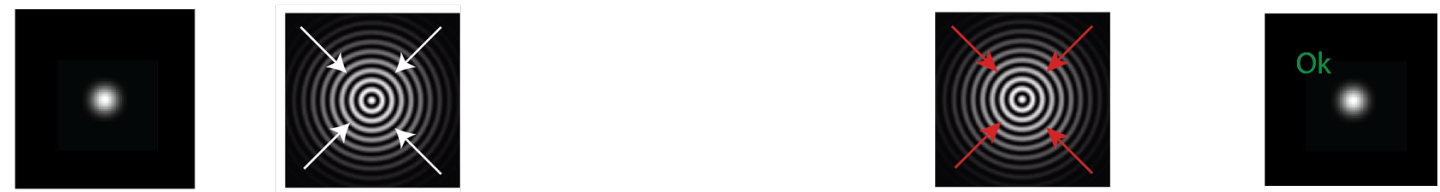

Fixation Baseline

VSO: Visual Stimulation Onset

$1-1.5 \mathrm{sec}$

$5 \mathrm{sec}$

$1-3 \mathrm{sec}$

CSO: Change Onset

Feedback

$0.2-0.8 \mathrm{sec}$

$0.5 \mathrm{sec}$

$\overrightarrow{\text { time }}$

B

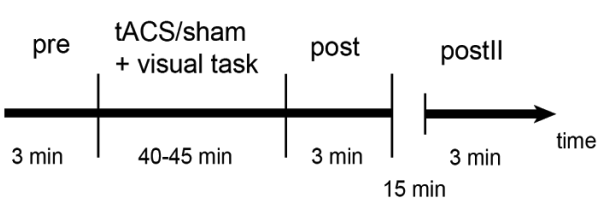

\section{C}

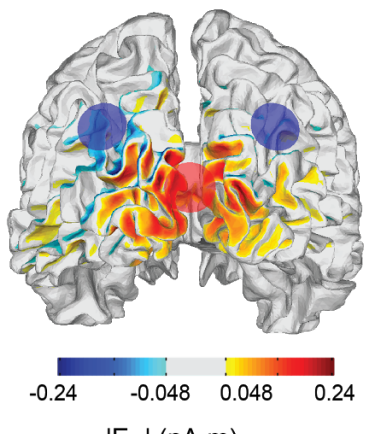

$\mathrm{D}$

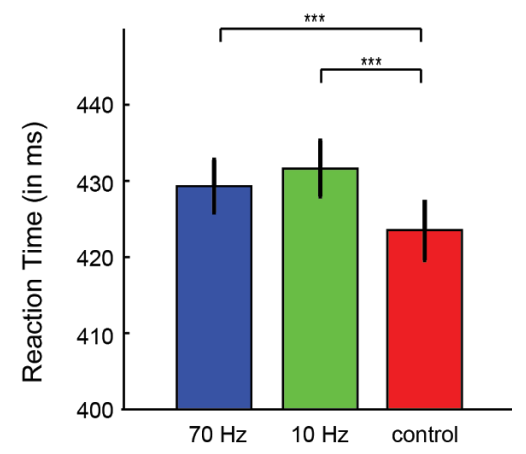

Figure 1. Experimental paradigm and behavioral responses. (A) Visual change-detection task. Subjects were instructed to report the change of speed of an unpredictable visual stimulus. The fixation period was followed by the presentation of a sine-wave grating moving inwards, centered at the fixation point. Bursts of tACS are delivered concurrently at the onset of visual stimulation (VSO) for $5 \mathrm{~s}$ in tACS sessions. At a random time between 1 and $3 \mathrm{~s}$, the velocity of the moving grating increases (CSO). Subjects reported change of speed by a keypress with right finger and received feedback OK/KO on correct detection (less than $0.8 \mathrm{~s}$ after CSO). (B) Experimental procedure. Each session starts and ends with 3 minutes of eyes open resting state (pre, post and postlI) during fixation. tACS/control intervals contained 240 trials of the visual task, adding up to $30 \mathrm{~min}$ of stimulation. (C) Current flow of the tACS using a multi-electrode optimized montage [19], revealing highest current flow in occipital cortex (stimulation electrodes located at PO3, PO4, Oz). (D) Reaction time in ms for the different stimulation protocols. 
A
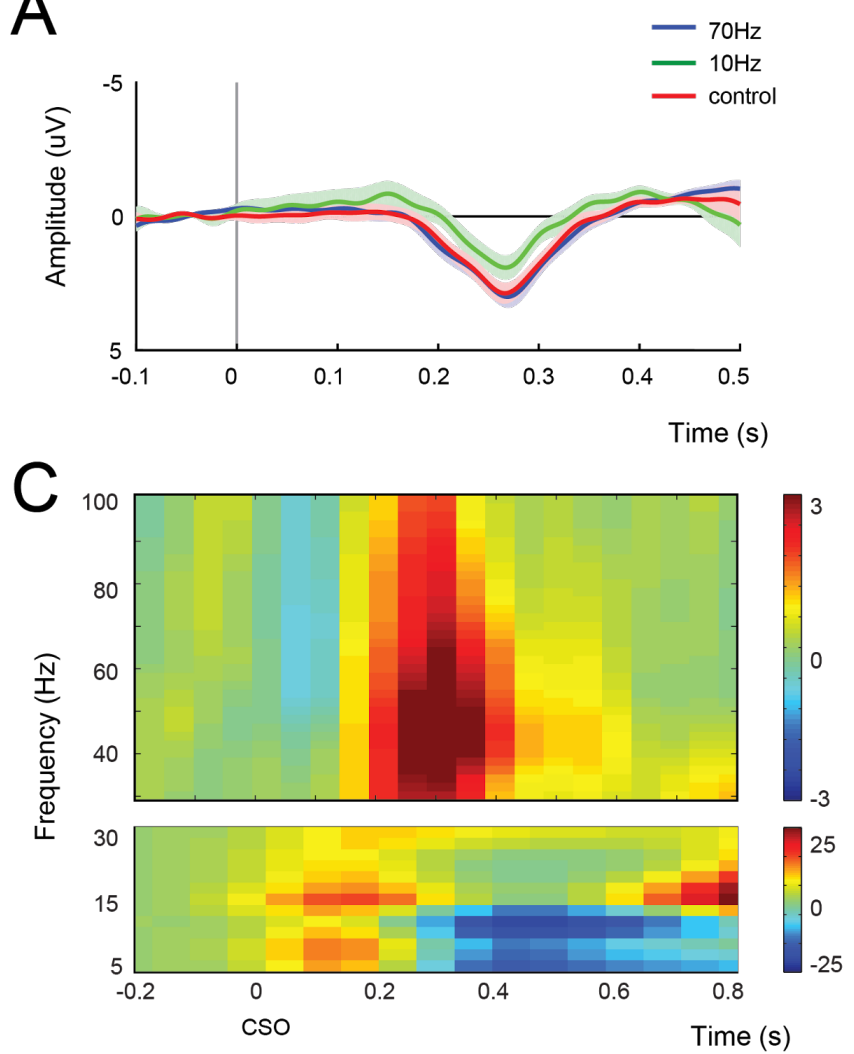

B

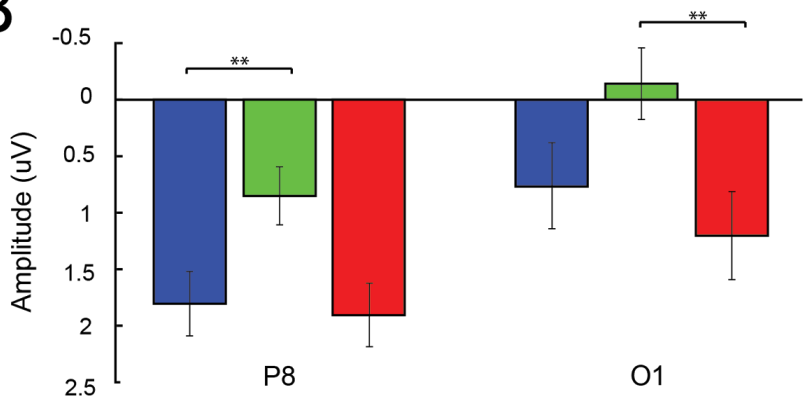

D

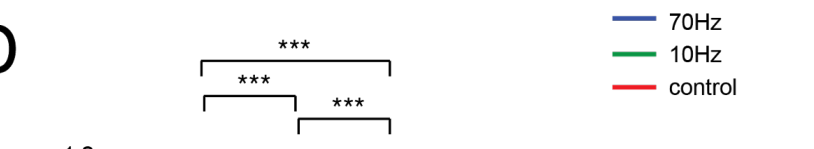

Figure 2. Visually induced responses in the EEG signals in time and power. (A) Event-related potential (ERP) associated to the CSO (baseline [-200 0] ms) for the different stimulation protocols at electrode P8 and (B) Average P300 amplitude at P8 and $\mathrm{O} 1$ electrodes. (C) TFR of the control sessions, expressed as a percentage of change with respect to baseline [-200 0]. (D) Percentage of change of incoherent $\alpha$ and $p$ power immediately after tACS bursts in occipital electrodes (0.25-1s after burst at 0102). Asterisks in A indicate statistical significance as assessed by pairwise analysis of GLMM factors $(p<0.001)$.
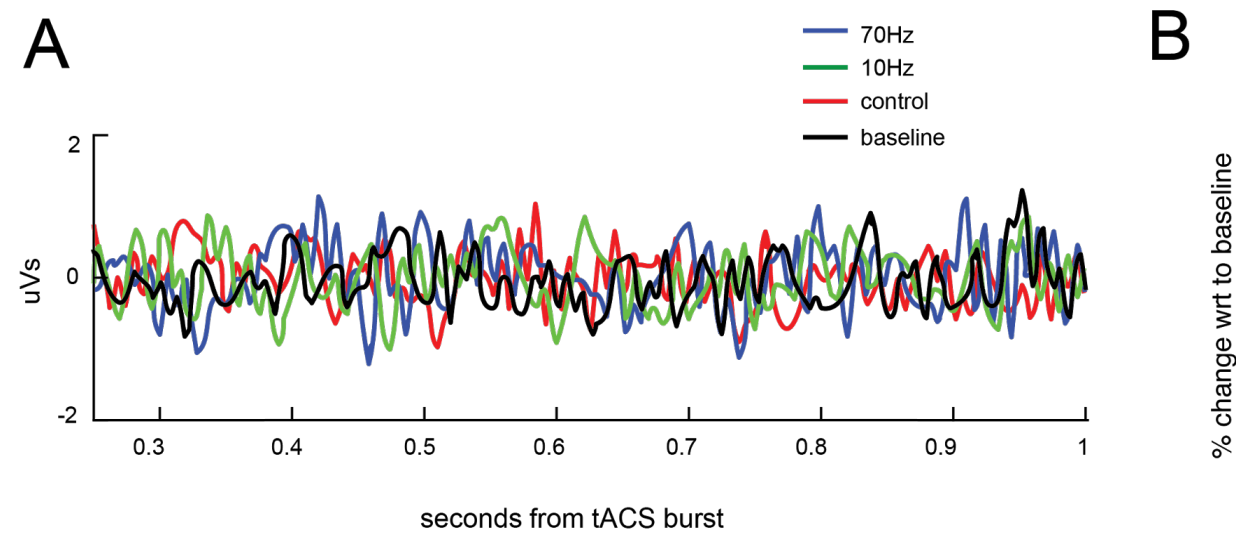

Figure 3. tACS Stimulation Related Potential and power change of EEG signals. (A) Stimulation Related Potential (SRP) associated to the tACS end-of-burst for a representative subject. Baseline is taken from the pre-EEG interval, see Figure $1 \mathrm{~A})$. (B) Percent of change in phase-locked $\gamma$ power at the onset of tACS bursts in occipital electrodes $(0.25-1.25 \mathrm{~s}$ after tACS burst at 0102). 
A

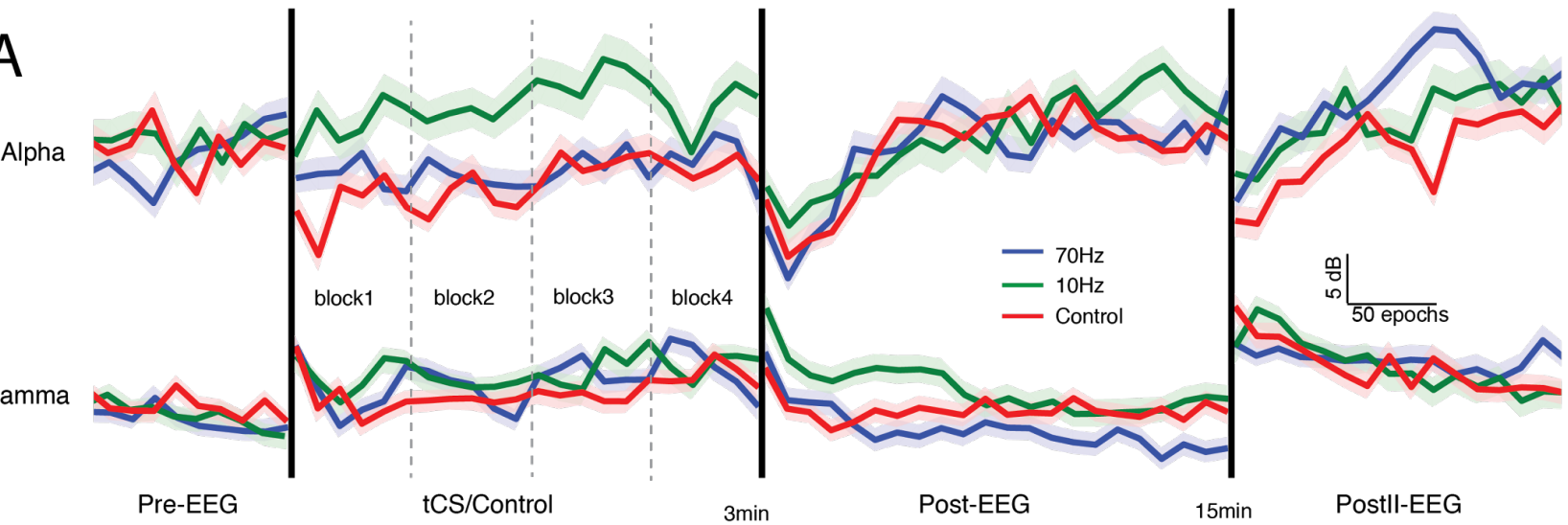

B

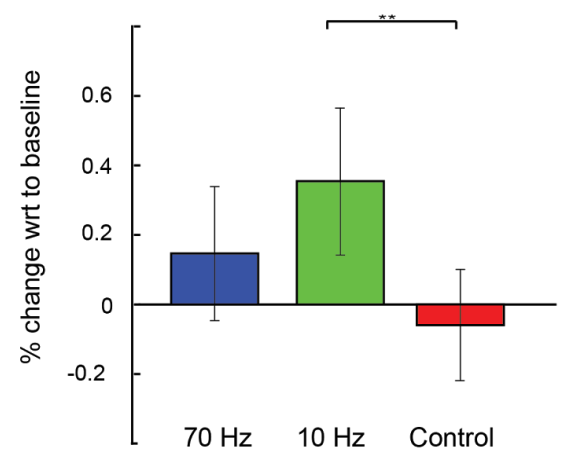

C

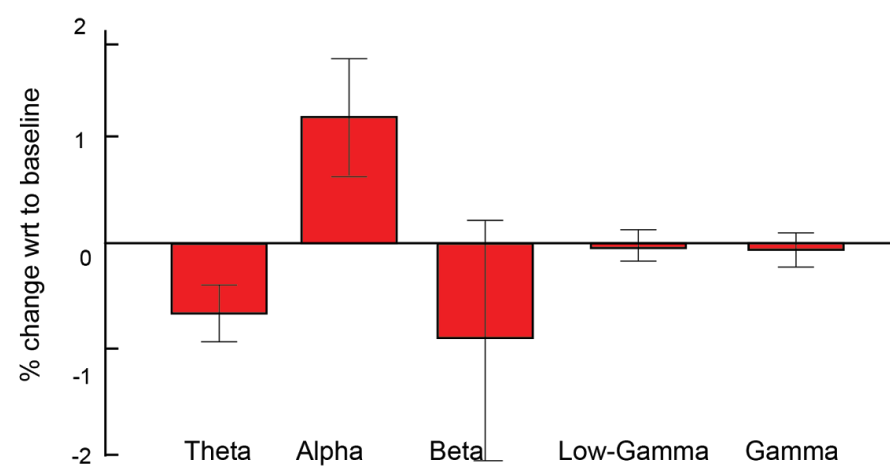

Figure 4. tACS after effects in power and signal complexity. A) Power change over time for 1-s epochs across for each experimental session. B) Change in LZW at $\gamma$ frequency at during the tCS condition at occipital electrodes. C) Complexity signature of the behavioral task in Control sessions. 


\section{REFERENCES}

1. Herrmann CS, Rach S, Neuling T, Strüber D. Transcranial alternating current stimulation: a review of the underlying mechanisms and modulation of cognitive processes. Front Hum Neurosci. 2013;7: 279.

doi:10.3389/fnhum.2013.00279

2. Vossen A, Gross J, Thut G. Alpha power increase after transcranial alternating current stimulation at alpha frequency (a-tACS) reflects plastic changes rather than entrainment. Brain Stimul. Elsevier Inc.; 2015;8: 499-508. doi:10.1016/j.brs.2014.12.004

3. Helfrich RF, Schneider TR, Rach S, Trautmann-Lengsfeld SA, Engel AK, Herrmann CS. Entrainment of brain oscillations by transcranial alternating current stimulation. Curr Biol. Elsevier Ltd; 2014;24: 333-339. doi:10.1016/j.cub.2013.12.041

4. Hoogenboom N, Schoffelen J-M, Oostenveld R, Fries P. Visually induced gamma-band activity predicts speed of change detection in humans. Neuroimage. Elsevier Inc.; 2010;51: 1162-7. doi:10.1016/j.neuroimage.2010.03.041

5. Michalareas G, Vezoli J, van Pelt S, Schoffelen JM, Kennedy H, Fries P. Alpha-Beta and Gamma Rhythms Subserve Feedback and Feedforward Influences among Human Visual Cortical Areas. Neuron. Elsevier Inc.; 2016;89: 384-397. doi:10.1016/j.neuron.2015.12.018

6. Fries P. Neuronal gamma-band synchronization as a fundamental process in cortical computation. Annu Rev Neurosci. 2009;32: 209-24. doi:10.1146/annurev.neuro.051508.135603

7. Clayton MS, Yeung N, Cohen Kadosh R. The roles of cortical oscillations in sustained attention. Trends Cogn Sci. Elsevier Ltd; 2015;19: 188-195. doi:10.1016/j.tics.2015.02.004

8. Uhlhaas PJ, Pipa G, Lima B, Melloni L, Neuenschwander S, Nikolic D, et al. Neural synchrony in cortical networks: history, concept and current status. Front Integr Neurosci. 2009;3: 1-19. doi:10.3389/neuro.07

9. Ruffini G. An algorithmic information theory of consciousness. Neurosci Conscious.

10. Gao RD, Peterson EJ, Voytek B. Field Potential Reflects the Balance of Synaptic Excitation and Inhibition. bioRxiv. 2016; 1-16. doi:10.1101/081125

11. Sejnowski TJ. Network Oscillations: Emerging Computational Principles. J Neurosci. 2006;26: 1673-1676. doi:10.1523/JNEUROSCI.3737-05d.2006

12. Abraham Lempel JZ. On the complexity of finite sequences. IEEE Trans Inf theory. 1976;22.

13. Ruffini G. Lempel-Ziv Complexity Reference. arXiv. 2017;

14. Cover TM, Thomas JA. Elements of Information Theory. Second Edi. Wiley-Interscience; 2006.

15. Santarnecchi E, Muller T, Rossi S, Sarkar A, Polizzotto NR, Rossi A, et al. Individual differences and specificity of prefrontal gamma frequency-tACS on fluid intelligence capabilities. Cortex. 2016;75: 33-43. doi:10.1016/j.cortex.2015.11.003

16. Miranda PC, Lomarev M, Hallett M. Modeling the current distribution during transcranial direct current stimulation. Clin Neurophysiol. 2006;117: 1623-1629. doi:10.1016/j.clinph.2006.04.009

17. Ruffini G, Wendling F, Merlet I, Molaee-Ardekani B, Mekonnen A, Salvador R, et al. Transcranial current brain stimulation (tCS): Models and technologies. IEEE Trans Neural Syst Rehabil Eng. 2013;21: 333-345. doi:10.1109/TNSRE.2012.2200046

18. Miranda PC, Mekonnen A, Salvador R, Ruffini G. The electric field in the cortex during transcranial current stimulation. Neuroimage. 2013;70: 48-58. doi:10.1016/j.neuroimage.2012.12.034

19. Ruffini G, Fox MD, Ripolles O, Miranda PC, Pascual-Leone A. Optimization of multifocal transcranial current stimulation for weighted cortical pattern targeting from realistic modeling of electric fields. Neuroimage. 2014;89: 216-225. doi:10.1016/j.neuroimage.2013.12.002

20. Fröhlich F, Sellers KK, Cordle AL. Targeting the neurophysiology of cognitive systems with transcranial alternating current stimulation. Expert Rev Neurother. 2015;15: 145-67. doi:10.1586/14737175.2015.992782

21. Antal A, Paulus W. Transcranial alternating current stimulation (tACS). Front Hum Neurosci. 2013;7: 1-4. doi:10.3389/fnhum.2013.00317

22. Lefaucheur J-P, Antal A, Ayache SS, Benninger DH, Brunelin J, Cogiamanian F, et al. Evidence-based guidelines on the therapeutic use of transcranial direct current stimulation (tDCS). 2017; doi:10.1016/j.clinph.2016.10.087

23. Polanía R, Paulus W, Nitsche MA. Noninvasively Decoding the Contents of Visual Working Memory in the Human Prefrontal Cortex within High-gamma Oscillatory Patterns. J Cogn Neurosci. 2011;24: 304-314. doi:10.1162/jocn

24. Laczó B, Antal A, Niebergall R, Treue S, Paulus W. Transcranial alternating stimulation in a high gamma frequency range applied over V1 improves contrast perception but does not modulate spatial attention. Brain Stimul. 2012;5: 484-491. doi:10.1016/j.brs.2011.08.008

25. Brignani D, Ruzzoli M, Mauri P, Miniussi C. Is Transcranial Alternating Current Stimulation Effective in Modulating Brain Oscillations? PLoS One. 2013;8. doi:10.1371/journal.pone.0056589

26. Thut G, Schyns PG, Gross J. Entrainment of perceptually relevant brain oscillations by non-invasive rhythmic stimulation of the human brain. Front Psychol. 2011;2: 1-10. doi:10.3389/fpsyg.2011.00170

27. Neuling T, Rach S, Herrmann CS, Miniussi C, Schwiedrzik CM. Orchestrating neuronal networks: sustained after- 
effects of transcranial alternating current stimulation depend upon brain states. 2013; doi:10.3389/fnhum.2013.00161

28. Krause B, Cohen Kadosh R. Not all brains are created equal: the relevance of individual differences in responsiveness to transcranial electrical stimulation. Front Syst Neurosci. 2014;8. doi:10.3389/fnsys.2014.00025

29. Schmidt SL, lyengar AK, Foulser AA, Boyle MR, Fröhlich F. Endogenous cortical oscillations constrain neuromodulation by weak electric fields. Brain Stimul. 2014;7: 878-889. doi:10.1016/j.brs.2014.07.033

30. Feurra M, Pasqualetti P, Bianco G, Santarnecchi E, Rossi A, Rossi S. State-Dependent Effects of Transcranial Oscillatory Currents on the Motor System: What You Think Matters. J Neurosci. 2013;33: 17483-17489. doi:10.1523/JNEUROSCI.1414-13.2013

31. Marshall L, Kirov R, Brade J, Mölle M, Born J. Transcranial electrical currents to probe EEG brain rhythms and memory consolidation during sleep in humans. PLoS One. 2011;6. doi:10.1371/journal.pone.0016905

32. Ruhnau P, Neuling T, Fuscá M, Herrmann CS, Demarchi G, Weisz N. Eyes wide shut: Transcranial alternating current stimulation drives alpha rhythm in a state dependent manner. Sci Rep. 2016;6: 27138. doi:10.1038/srep27138

33. Zaehle T, Rach S, Herrmann CS. Transcranial Alternating Current Stimulation Enhances Individual Alpha Activity in Human EEG. PLoS One. 2010;5: 1-7. doi:10.1371/journal.pone.0013766

34. Herrmann CS, Murray MM, Ionta S, Hutt A, Lefebvre J. Shaping Intrinsic Neural Oscillations with Periodic Stimulation. J Neurosci Off J Soc Neurosci. 2016;36: 5328-5337. doi:10.1523/JNEUROSCI.0236-16.2016

35. Hoogenboom N, Schoffelen J-M, Oostenveld R, Parkes LM, Fries P. Localizing human visual gamma-band activity in frequency, time and space. Neuroimage. 2006;29: 764-73. doi:10.1016/j.neuroimage.2005.08.043

36. Muthukumaraswamy SD. High-frequency brain activity and muscle artifacts in MEG/EEG: a review and recommendations. Front Hum Neurosci. 2013;7: 138. doi:10.3389/fnhum.2013.00138

37. Li Y, Tong S, Liu D, Gai Y, Wang X, Wang J, et al. Abnormal EEG complexity in patients with schizophrenia and depression. Clin Neurophysiol. 2008;119: 1232-1241. doi:10.1016/j.clinph.2008.01.104

38. Casali AG, Gosseries O, Rosanova M, Boly M, Sarasso S, Casali KR, et al. A Theoretically Based Index of Consciousness Independent of Sensory Processing and Behavior. Sci Transl Med. 2013;5: 198ra105-198ra105. doi:10.1126/scitranslmed.3006294

39. Perez AF, Ibor MIL, Turrero A, Santos JM, Moron MD, Hornero R, et al. Lempel-Ziv complexity in schizophrenia: A MEG study. Clin Neurophysiol. 2011;122.

40. Méndez MA, Zuluaga P, Hornero R, Gómez C, Escudero J, Rodríguez-Palancas A, et al. Complexity analysis of spontaneous brain activity: effects of depression and antidepressant treatment. J Psychopharmacol. 2012;26: 636643. doi:10.1177/0269881111408966

41. Gomez C, Hornero R. Entropy and Complexity Analyses in Alzheimer's Disease: An MEG Study. Open Biomed Eng J. 2010;4: 223-235. doi:10.2174/1874120701004010223

42. Fernández A, Zuluaga P, Abásolo D, Gómez C, Serra A, Méndez MA, et al. Brain oscillatory complexity across the life span. Clin Neurophysiol. 2012;123: 2154-2162. doi:10.1016/j.clinph.2012.04.025

43. Schartner MM, Carhart-Harris RL, Barrett AB, Seth AK, Muthukumaraswamy SD. Increased spontaneous MEG signal diversity for psychoactive doses of ketamine, LSD and psilocybin. Sci Rep. 2017;7: 46421. doi:10.1038/srep46421

44. Handy TC. Basic principles of ERP quantification. Event-related potentials: A methods handbook. 2005.

45. Molaee-Ardekani B, Márquez-Ruiz J, Merlet I, Leal-Campanario R, Es Gruart A, Sanchez-Campusano R, et al. Effects of transcranial Direct Current Stimulation (tDCS) on cortical activity: A computational modeling study. Effects of transcranial Direct Current Stimulation (tDCS) on cortical activity: A computational modeling study. Brain Stimul. Elsevier; 2013;6: 25-39. Available: http://www.hal.inserm.fr/inserm-00664021

46. Strüber D, Rach S, Neuling T, Herrmann CS, Soekadar SR, Sun H, et al. On the possible role of stimulation duration for after-effects of transcranial alternating current stimulation. 2015; doi:10.3389/fncel.2015.00311

47. Kasten FH, Dowsett J, Herrmann CS. Sustained aftereffect of $\alpha$-tACS lasts up to 70 minutes after stimulation. Front Hum Neurosci. 2016;10: Article 245. doi:10.3389/fnhum.2016.00245

48. Sliwinska MW, Vitello S, Devlin JT. Transcranial Magnetic Stimulation for Investigating Causal Brain-behavioral Relationships and their Time Course Video Link. J Vis Exp. 5173;3791. doi:10.3791/51735

49. Kasten FH, Herrmann CS. Transcranial Alternating Current Stimulation (tACS) Enhances Mental Rotation Performance during and after Stimulation. Front Hum Neurosci. 2017;11. doi:10.3389/fnhum.2017.00002

50. Abásolo D, Simons S, Morgado da Silva R, Tononi G, Vyazovskiy V V. Lempel-Ziv complexity of cortical activity during sleep and waking in rats. J Neurophysiol. 2015;113: 2742-2752. doi:10.1152/jn.00575.2014

51. Kok A. Event-related-potential (ERP) reflections of mental resources: A review and synthesis. Biological Psychology. 1997. pp. 19-56. doi:10.1016/S0301-0511(96)05221-0

52. Ramchurn A, de Fockert JW, Mason L, Darling S, Bunce D. Intraindividual reaction time variability affects P300 amplitude rather than latency. Front Hum Neurosci. 2014;8. doi:10.3389/fnhum.2014.00557

53. Antal A, Kincses TZ, Nitsche MA, Bartfai O, Paulus W. Excitability changes induced in the human primary visual cortex by transcranial direct current stimulation: direct electrophysiological evidence. Invest Ophthalmol Vis Sci. 2004;45: 702-707. doi:10.1167/iovs.03-0688 
bioRxiv preprint doi: https://doi.org/10.1101/198788; this version posted October 5, 2017. The copyright holder for this preprint (which was

not certified by peer review) is the author/funder, who has granted bioRxiv a license to display the preprint in perpetuity. It is made available under aCC-BY 4.0 International license.

54. De Taeye L, Vonck K, van Bochove M, Boon P, Van Roost D, Mollet L, et al. The P3 Event-Related Potential is a Biomarker for the Efficacy of Vagus Nerve Stimulation in Patients with Epilepsy. Neurotherapeutics. 2014;11: 612622. doi:10.1007/s13311-014-0272-3

55. Klimesch W, Sauseng P, Hanslmayr S. EEG alpha oscillations: the inhibition-timing hypothesis. Brain Res Rev. 2007;53: 63-88. doi:10.1016/j.brainresrev.2006.06.003

56. Stein A Von, Chiang C, Ko P. Top-down processing mediated by interareal synchronization. 2000;

57. Castellano M, Plöchl M, Vicente R, Pipa G. Neuronal oscillations form parietal/frontal networks during contour integration. Front Integr Neurosci. 2014;8: 1-13. doi:10.3389/fnint.2014.00064

58. Brunoni AR, Amadera J, Berbel B, Volz MS, Rizzerio BG, Fregni F. A systematic review on reporting and assessment of adverse effects associated with transcranial direct current stimulation. Int J Neuropsychopharmacol. 2011;14: 1133-1145. doi:10.1017/S1461145710001690

59. Poreisz C, Boros K, Antal A, Paulus W. Safety aspects of transcranial direct current stimulation concerning healthy subjects and patients. Brain Res Bull. 2007;72: 208-214. doi:10.1016/j.brainresbull.2007.01.004

60. Delorme A, Makeig S. EEGLAB: an open source toolbox for analysis of single-trial EEG dynamics including independent component analysis. J Neurosci Methods. 2004;134: 9-21. doi:10.1016/j.jneumeth.2003.10.009

61. Oostenveld R, Fries P, Maris E, Schoffelen J-M. FieldTrip: Open source software for advanced analysis of MEG, EEG, and invasive electrophysiological data. Comput Intell Neurosci. 2011;2011: 156869. doi:10.1155/2011/156869

62. Romero Lauro LJ, Rosanova M, Mattavelli G, Convento S, Pisoni A, Opitz A, et al. TDCS increases cortical excitability: Direct evidence from TMS-EEG. Cortex. Elsevier Ltd; 2014;58: 99-111. doi:10.1016/j.cortex.2014.05.003 